\title{
Confinement and Stability of a Crystalline Beam
}

?

JUI.

$O s+1$

\author{
Alessandro G. Ruggiero
}

May 10, 1993

\section{ALTERNATING GRADIENT SYNCHROTRON DEPARTMENT}

\section{BROOKHAVEN NATIONAL LABORATORY}

UPTON, LONG ISLAND, NEW YORK 11973

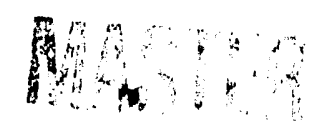

BISTRIBUTIOA of This Document is untimires 


\section{DISCLAIMER}

This report was prepared as an account of work sponsored by the United States Government. Neither the United States nor the United States Department of Energy, nor any of their employees, nor any of their contractors, subcontractors, or their employees, makes any warranty, express or implied, or assumes any legal liability or responsibility for the accuracy, completeness, or usefulness of any information, apparatus, product or process disclosed, or represents that its use would not infringe privately owned rights. 


\section{Abstract}

This technical report defines and describes a Crystalline Beam. This is an ordered S._. te of matter made of electrically charged ions which are moving together in a storage ring with very high density and small velocity spread. In particular, the paper analyses the requirements for the confinement and the stability of the Beam. It is demonstrated that a storage ring made of one circular weak-focusing magnet, similar to a Betatron, is the most cuitable for the confinement and stability of the Crystalline Beam. The disruptive effects of arift insertions have also been investigated. Requirements on final densities and velocity spreads are also calculated and reported. A matrix formalism is developed for the design of the storage ring. The important issue of the disruption caused by the curvature of the closed trajectory is not here discussed; it is the subject of a subsequent paper.

* Work performed under the auspices of the U.S. Department of Energy. 


\section{Introduction}

A Crystalline Beam is an ensemble of charged particles, all identical to each other, with the same electric charge $Q e$ and mass at rest $A m$, where $e$ is the electron charge and $m$ the proton mass at rest. Thu charge state $Q$ and mass number $A$ are integer. Particles are treated point-like, with no internal structure. Only interaction among each other is the electromagnetic interaction. It is assumed that there is an equilibrium configuration where particles occupy a rigid position with respect to each other while all together move in one direction. Particles are allowed to oscillate around their equilibrium positions as long as the amplitude of the oscillations is small.

A very cold state of matter made of ions circulating in a storage ring with high density and very small velocity spread can be obtained only with powerful cooling techniques. The requirement on the cooling rates is not the subject of this report.

The interest for experimentally demonstrating and controlling a Crystalline Beam is mainly due to the quest for a beam of ions where particles are essentially screened from the interaction of each other electromagnetic, thus virtually removing the limitations due to space-charge forces and intrabeam scattering. It is only under these conditions inat higher beam densities may be hoped to be obtained. Applications would be various, mostly in the field of particle accelerator technology, for instance in colliders where high luminosity performance is required.

This topic of research is relatively recent [1]. A very important workshop [2] on the subject was held at Werheim in West Germany on October 1988 and was attended by international participation. A proposal [3] has been made more recently to the INFN to huild a small heavy-ion storage ring at the Laboratori Nazionali di Legnaro, near Padua in Italy, for the experimental demonstration of a Crystalline Beam. In principle the project (CRYSTAL) has been approved, provided that the problem of determining the optimal magnet layout of the storage ring has been solved. This report addresses specifically this issue.

The magnet lattice of the storage ring has to be chosen to provide stability of motion at both extremes of the beam configuration: (i) at the early stage, when the beam is diluted and space-charge forces can be neglected, and (ii) at the end of cooling when space charge forces have increased considerably and when the final state has been reached. Our goal is to determine design prescription of the lattice suitable for stability at both extremes. We shall not concern ourselves here on the modality for the transition between the two states. More in particular, we shall have to define clearly the properties of the Crystalline Beam, since those of the initial state are already well known.

There are two separate issues: one related to the confinement and the other to the stability of a Crystalline Beam. Antecedent to both is the search for an equilibrium configuration, that is the determination of an equilibrium distribution where ions occupy a rigid

position with respect to each other and are effectively screened from each other. It may be required to maintained this distribution with some external forces, the determination of which is the task of the confinement issue. Moreover, it will be important to determine whether and under which conditions the equilibrium distribution is stable, which is the task of the stability issue. It is obvious that the magnet lattice of the storage ring has to provide simultaneously the confinement and the stability of the Crystalline Beam. 
Confinement and stability questions are best described with the rectangular and infinite Crystalline Beam model which is introduced in Section 2. We consider next the case of a more realistic cylindrical heam, infinitely long in the longitudinal direction, hut having finite transverse dimensions. Section 3 detines this beam. Section 4 discusses the requirements for the longitudinal confinement and stability. Section 5 and 7 examine respectively the continement and stability in the plane transverse to the direction of motion. It is seen that, in order to keep the beam confined transversely, a circular magnet with a field profile providing focusing simultaneously in both transverse directions is required. The magnet could be made similar to a Betatron as shown in Section 6. The field profile has also to be chosen so to maintain beam stability against transverse oscillations. Since the external restoring force is linear with the particle transverse displacement from the heam axis, it is also important that the equilibrium configuration places particles in such a way that the resulting space-charge forces are also linear.

Section 8 defines quantitatively the limits of the beam spreads in momenta as evidence of crystallization. Section 9 is an analysis of the effects introduced by the insertions of drifts. The resulting storage ring lattice is to show stability at the two extremes: when the space charge is ignored and for the final state of the Crystalline Beam. This is described in Section 10. Finally an example is given in Section 11.

\section{The Rectangular Crystalline Beam Model}

Let us begin by considering a beam made of an infinitely large number of particles. They all have the same velocity $v$, with magnitude $v=\beta c$, where $c$ is the light velocity and $\beta$ the relativistic velocity factor. Similarly $\gamma=1 / \sqrt{1-\beta^{2}}$ is the relativistic energy factor. Particles are uniformly distributed in the three-dimensional physical space, extending to infinity in all three dimensions. They are equally spaced from each other, sitting at the knots of a rectangular grid with step-size $\lambda_{\|}$along the direction of motion and $\lambda_{\perp}$ in the plane transverse to it as shown in Figure 1.

The location of a particle in the Crystalline Beam can be determined with a threedimensional vector $i$ having integers as components: $\left(i_{1}, i_{2}, i_{3}\right)$. Each integer denotes the distance in number of steps along one direction (either 1, 2 or 3) from an origin () defined at (0, (), ()). The integer indices range from $-\infty(0)+\infty$. If, along one direction, a particle has index $i$ and another index $j$, the $i$-th particle will lead the $j$-th particle in that direction if $i>j$ and will trail if $i<j$. The $i$ - th particle, that is the one which is associated to the position vector $i$, can also be associated to a location vector $\boldsymbol{r}_{\boldsymbol{i}}$ which is directed from the origin () to the particle position:

$$
r_{i}=\lambda_{\perp}\left(i_{1}, i_{2},()\right)+\lambda_{\|}\left(0,0, i_{3}\right)=r_{(0 i}
$$

Because the particles move with the same velecity and in the same direction, which we have taken as the direction along the axis 3 , it is possible to operate a transtormation $(0)$ the system where they are at rest. In this frame, particles generate and experience only electric fields.

In the following, though expressions may he given either in the laboratory or in the rest frame, single paramelers, like beam dimensions, are always given in the laboratory frame. 


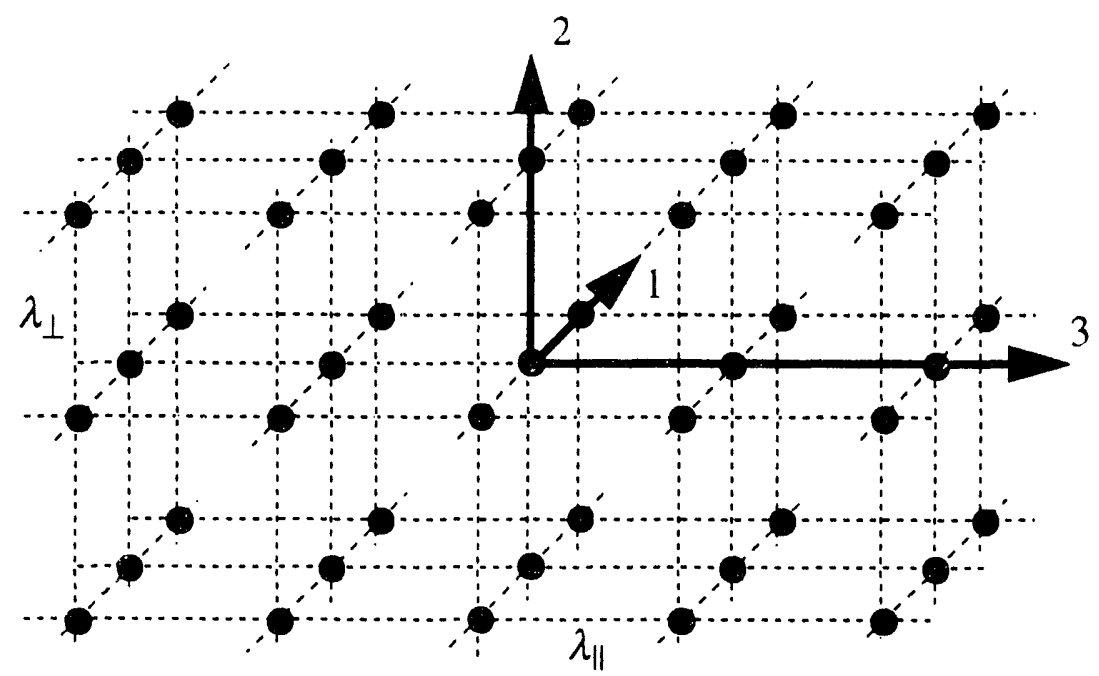

Fig. 1. A Rectangular Crystalline Beam

Whereas the dimensions along the axis 1 and 2, transverse to the main direction of motion, remain unchanged, there is a dilation of the longitudinal dimension, so that in the frame at rest $i=\left(i_{1}, i_{2}, \gamma i_{3}\right)$.

The electric field experienced by the $i$ - th particle is the sum of all the fields generated by the other particles, that is

$$
E_{i}=Q e \Sigma_{j \neq i} \frac{r_{i}-r_{j}}{\left|r_{i}-r_{j}\right|^{3}}
$$

Because of the symmetric arrangement of the particles, the electric field is the same for all particles and identically equal to zero. Thus there is no interaction between particles; that is they are completely screened from each other. This configuration is in equilibrium and does not need external means to be confined. Once they acquire their position, particles will remain at rest indefinitely.

To determine whether the equilibrium configuration is stable we add a perturbation of motion to the $i$ - th particle, leaving the position of all other particles unchanged. The perturbation is in the longitudinal direction, which is the direction along the axis 3 , that is the direction of motion. We take $\boldsymbol{r}_{i}=\boldsymbol{r}_{0 i}+\lambda_{\|} \gamma \boldsymbol{u}_{i}$, with $\boldsymbol{r}_{0 i}$ given by Eq. (1) and $\boldsymbol{u}_{i}=\left(1,0, u_{i}\right)$, in the expression for the electric field $E_{i}$ acting on the $i$ - th particle. The perturbation is small, that is $u_{i}<<1$. Again because of symmetry, with a simple translation of the axis origin $O$, we can take the $\boldsymbol{i}$ - th particle coinciding with the one at the origin, that is $\boldsymbol{i}=\boldsymbol{\theta}=$ $(0,0,0)$. After linearizing with respect to $u_{i}$ we find that only the longitudinal component of the field is not identically vanishing

$$
E_{3 i}=-Q_{e} w g_{\|}(w) u_{i} / \lambda_{\perp}^{2}
$$

where $w=\lambda_{\|} \gamma / \lambda_{\perp}$ is the compactness parameter, and 


$$
\xi_{\|}(w)=\Sigma_{j \neq 0} \frac{2 w^{2} j_{3}^{2}-j_{1}^{2}-j_{2}^{2}}{\left(j_{1}^{2}+j_{2}^{2}+w^{2} j_{3}^{2}\right)^{5 / 2}}
$$

is the longitudinal response function plotted in Figure 2. In particular $g_{\|}(1)=0$.

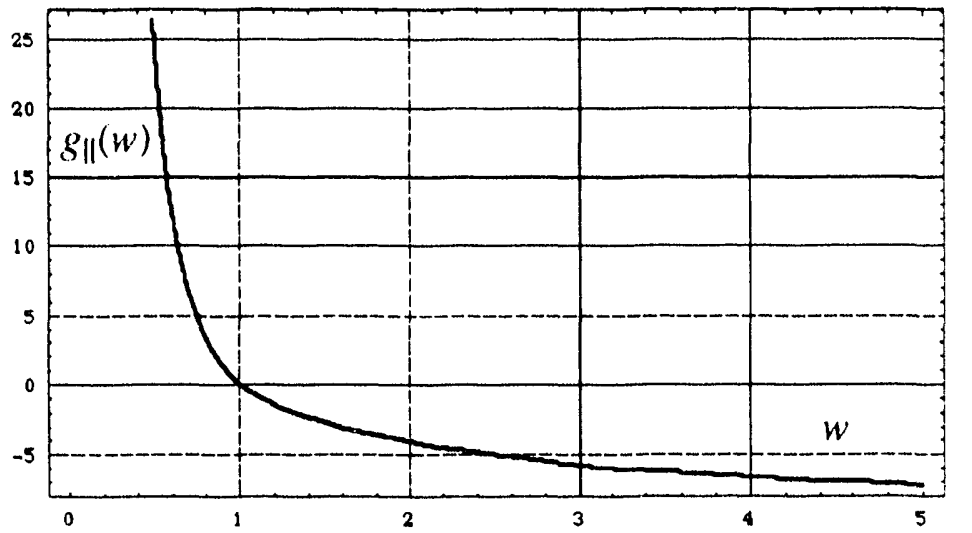

Fig. 2. The Longitudinal Response Function $g_{\|}(w)$ versus $w$

We can also write the equation of motion for the $i$ - th particle as follows

$$
\begin{aligned}
m A \gamma \lambda_{\|} \ddot{u}_{i} & =Q_{e} E_{3 i} \\
\ddot{u}_{i} & =-\Omega_{\|}^{2} u_{i}
\end{aligned}
$$

where

$$
\Omega_{\|}^{2}=\frac{Q^{2} e^{2} g_{\|}(w)}{\lambda_{\perp}^{3} \gamma^{2} m A}
$$

and $\Omega_{\|}$is the longitudinal angular response frequency of the Crystalline Beam (already corrected for the transformation to the laboratory frame).

Similarly, we can estimate the stability of the equilibrium configuration against a perturbation of motion occurring in the plane transverse to the direction of motion, that is either along the axis 1 or the axis 2 . The transverse angular response frequency $\Omega_{\perp}$ of the Crystalline Beam is then given by (after correction for the transformation to the laboratory trame)

$$
\Omega_{\perp}^{2}=\frac{Q^{2} e^{2} g_{\perp}(w)}{\lambda_{\perp}^{3} \gamma^{2} m A}
$$

where $g_{\perp}(w)=-g_{\|}(w) / 2$ is the transverse response function.

The two response fiequencies have different dependence with the aspect ration $w$ of the Crystalline Beam. For the motion to be stable $\Omega_{\|}{ }^{2}$ and $\Omega_{\perp}{ }^{2}$ are $t$ o be positive. This cannot be satistied at the same time since the response functions $g_{\|}(w)$ and $g_{\perp}(w)$ have opposite signs. In order for the beam to be stable against a longitudinal perturbation one 
needs $w<1$, that is the beam has to be more compact longitudinally. For the beam to be stable against a transverse perturbation $w>1$ is required, that is the beam is more compact transversely. As a particular case, which is difficult to maintain and to control in practice, both frequencies vanish identically if $w=1$.

(a)

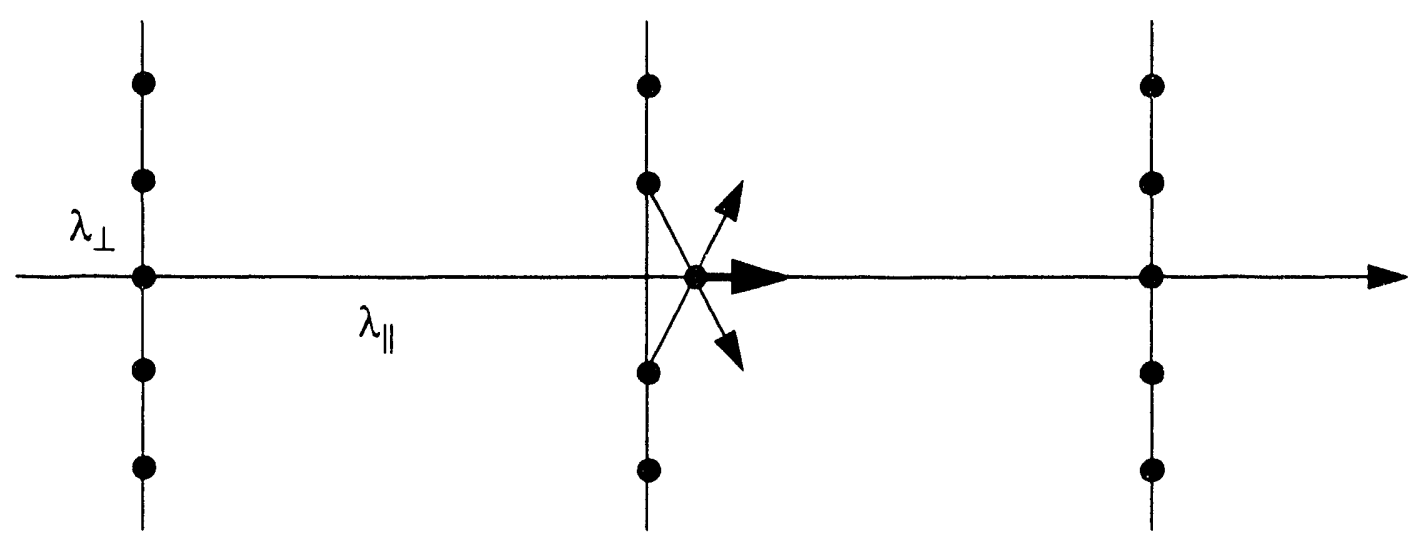

(b)

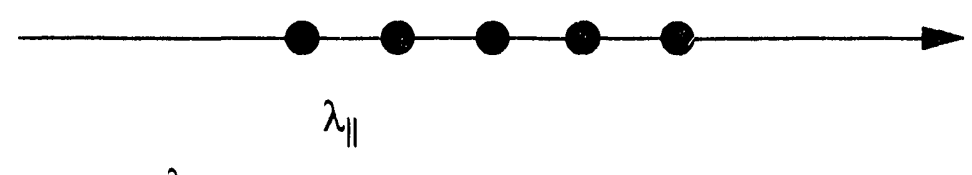

$\lambda_{\perp}$
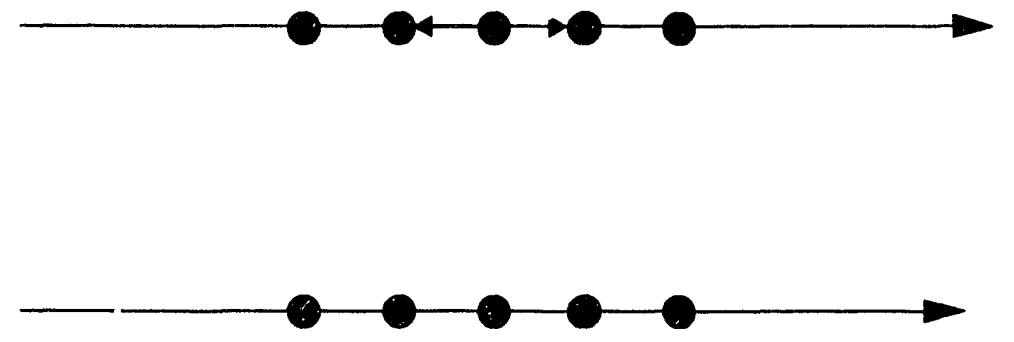

Fig. 3. (a) Longitudinally Diluted Beam. (b) Longitudinally Compact Beam

There is no need for beam confinement along the direction where the beam configuration is stable. Confinement with external means is required along the direction(s) where the beam is unstable. A practical example is given by the case of a longitudinally compact beam $(w<1)$ where the longitudinal motion is stable and that in the transverse plane is unstable, requiring therefore confinement in the transverse plane with an external restoring force.

It is relatively easy to explain why a rectangular Crystalline Beam has to be more compact longitudinally to maintain stability in the longitudinal direction. This can be seen by inspecting the following Figures $3 a, b$. Figure $3 a$ describes a longitudinally diluted beam with $\lambda_{\|}>\lambda_{\perp}$. In this case, the particle with the perturbation is affected only by 
those immediately above and helow, the others on the sides are too far away to cause any effect. The resulting field is longitudinal and has the effect to push the particle away.

Figure 3 h describes a longitudinally compace beam with $\lambda_{\|}<\lambda_{\perp}$. The particle on which the motion perturbation is applied is affected only by those immediately on the sides, the others ahove and below are too far away to cause any effect. The resulting field is also longitudinal, but this time has the effect 10 push the particle hack to its original !ocation.

\section{The Cylindrical Crystalline Beam Model}

Consider now a Crystalline Beam of infinite length along the direction of motion, but with tinite transverse dimensions. We shall continue to determine the position of a particle with a vector $i$ of three components having integer values, that is $i=\left(i_{1}, i_{2}, i_{3}\right)$. All particles move with the same velocity $v$, as before, to allow an easy relativistic transformation to the rest frame. The direction of motion is taken again along the axis 3 .

To preserve an equilibrium contiguration, particles are uniformly distributed along the longitudinal direction 3 where they are also equally spaced by a distance $\lambda_{\|}$. The vector position of the $i$ - th particle in the frame at rest can be written as

$$
r_{i}=r_{\perp i}+r_{\| i}
$$

where $r_{\| i}=\gamma \lambda_{\|}\left(0,0, i_{3}\right)$.

No confinement should be required along the longitudinal direction. The transverse motion of the particle is kept contined with the aid of an external halancing force. If we lake this to be linear with the transverse particle coordinates, we expect the heam to tatke in general an elliptic cross-section.

We describe a Crystalline Beam with an equilibrium configuration where particles are located on concentric shells stretching longitudinally and having elliptic cross-sections of the same aspect ratio (see Figure 4). The longitudinal distribution is uniform, whereas, transversely, particles are distributed between a number $n_{s}$ of shells of elliptic crosssection of varying semi-axis $b_{1}$ and $b_{2}$, the former along the axis $I$ and the second along the axis 2. For all shells, the aspect ratio $\eta=b_{2} / h_{1}$ is the same. Each shell is made of $n / h$ particles equally spaced and placed at locations which are ohtained from one another with an azimuthal rotation of a multiple of the angle $\theta=2 \pi / n_{h}$

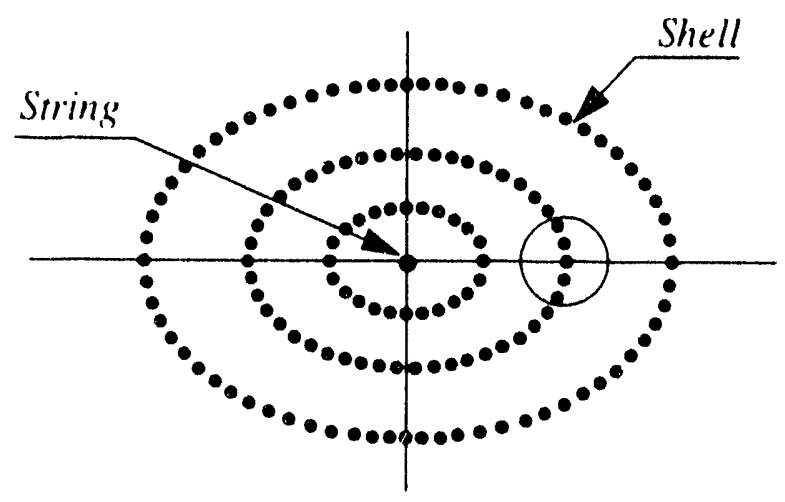

Fig. 4. Transverse Cross-section of a Cylindrical Crystalline Beam 
Thus the longitudinal integer index $i_{3}$ still ranges from $-\infty$ to $+\infty$ and describes the longitudinal position of the $i$-th particle within the Crystalline Beam. The second index $i_{2}$, varying from 1 to $n_{l}$, describes the azimuthal position of the particle. Finally the first index $i_{1}$ (simply $i$ ), ranging from 0 to $n_{s}$, assigns a particle to a shell. The innermost shell with $i_{1}=0$ is the string, that is a shell with vanishing semi-axis, $b_{1}=b_{2}=0$. The string also coincides with the longitudinal axis 3, that is the beam axis itself. We can then write

$$
r_{\perp i}=\left[b_{1 i} \cos \left(\theta_{0 i}+\theta i_{2}\right), b_{2 i} \sin \left(\theta_{0 i}+\theta i_{2}\right), 0\right]=r_{0 \perp i}
$$

where the angle cffset $6_{0 i}$ may vary from shell to shell.

In general a Crystalline Beam with elliptic cross-section is described by the following parameters:

--- the number $n_{s}$ of shells,

--- the number $n_{h}$ of particles per shell, which determines also the separation angle $\theta$,

--- the offset angles $\theta_{0 i}$ for all shells $\left(i=1, \ldots, n_{s}\right)$, and

-.. the aspect ratio $\eta$ of the cross-section.

The actual semi-axis of the shells are determined as shown below.

It can be easily seen, with symmetry considerations, that the longitudinal component of the electric field acting on any particle banishes identically, whereas the transverse components of the field are in general different from zero. Actually they are zero on the beam axis and increase in amplitude toward the edge of the beam. The transverse component of the electric field is always directed away from the beam axis. The amplitude and direction of the electric fieid is independent of the longitudinal position of the particle within the beam.

We assume that there is an equilibrium configuration where particles are located at such positions, transversely with regards to the beam axis, so that the components of the electric field vary linearly with the position of the particle. That is

$$
\begin{aligned}
E_{\perp \mathbf{i}} & =Q e \Sigma_{j \neq i} \frac{r_{\perp i}-r_{\perp j}}{\left|r_{i}-r_{j}\right|^{3}} \\
& =\left(k_{1} r_{1 i}, k_{2} r_{2 i}\right)
\end{aligned}
$$

where $r_{1 i}$ denotes the location along the axis 1 , and $r_{2 i}$ that along the axis 2 .

In the limit of a large number $N$ of particles [4]

$$
k_{1,2}=\frac{2 Q_{e}(N / L)}{\gamma \alpha b_{1,2}{ }^{2}}
$$

where $(N / L)$ is the number of particles per unit length, that is, $N$ is the number of particles 
in a length $L$ (laboratory frame) of the beam. The expression above still applies to the frame where the heam is at rest. Finally $\alpha$ is the following function of $\eta=b_{2} / b_{1}$ with $b_{1}$ and $b_{2}$ the beam semi-axis, respectively along the direction 1 and 2 ,

$$
2 \pi \alpha(\eta)=\int_{-\pi}^{+\pi} \sqrt{\frac{\sin ^{2} \tau+\eta^{2} \cos ^{2} \tau}{\cos ^{2} \tau+\eta^{2} \sin ^{2} \tau}} d \tau
$$

If $\eta=1$ (round beam) then $\alpha=1$. The $\alpha$ function is plotted in Figure 5 . It is easily seen that

$$
\alpha(1 / \eta)=\alpha(\eta)
$$

To preserve the linearity of the electric field generated by the beam, the values of the semi-axis of the $i$-th shell are, for $n_{h} \gg 1$,

$$
b_{1 i}=b_{1} \sqrt{i / n_{s}} \text { and } b_{2 i}=b_{2} \sqrt{i / n_{s}}
$$

Thus $b_{1}$ and $b_{2}$ are also the semi-axis of the outermost shell $\left(i_{1}=n_{s}\right)$.

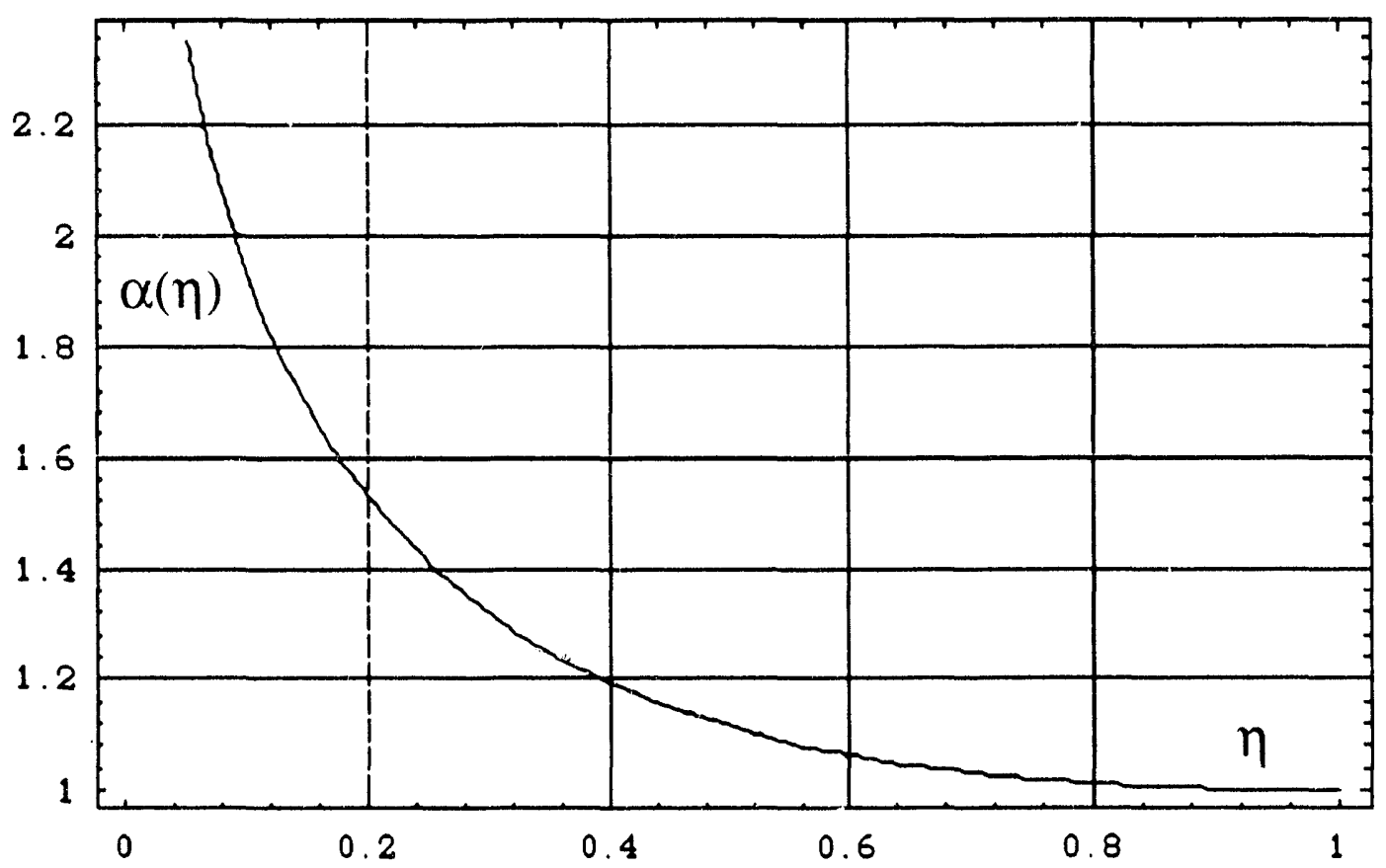

Fig. 5 The $\alpha(\eta)$ function 


\section{Longitudinal Confinement and Stability of the Cylindrical Beam}

Let us hegin by determining the longitudinal stability of the cylindrical Crystalline Beam. At this purpose, we add a longitudinal perturbation of amount $\gamma \lambda_{\|} u_{i}$ (in the rest frame) to the motion of the $i$ - th particle. We take $u_{i} \ll<1$ and linearize the expression of the electric field acting on the particle itself.

To simplify our analysis we shall examine the special case of a round Crystalline Beam for which $b_{1}=b_{2}=b$ and $\eta=1$ so that also $\alpha(\eta)=1$. For this case it can be shown that again only the longitudinal component of the field does not vanish. For reasons of symmetry, it is also easily seen that the longitudinal field does not depend on the actual location of the particle on a shell, so that, without violating generality, we can take $i_{2}=i_{3}$ $=0$ and iet $i=(i, 0,())$. We obtain

$$
E_{3 i}=-Q e w g_{\|}(w) u_{i} n_{s} / b^{2}
$$

where now

$$
w^{2}=\gamma^{2} \lambda_{\|}^{2} n_{s} / b^{2}
$$

and

$$
g_{\|}(w)=\Sigma_{j \neq i} \frac{2 w^{2} j_{3}{ }^{2}-i-j+2 \sqrt{i j} \cos \left(\theta_{0 i}-\theta_{0 j}+\theta j_{2}\right)}{\left[i+j-2 \sqrt{i j} \cos \left(\theta_{0 i}-\theta_{0 j}+\theta j_{2}\right)+w^{2} j_{3}{ }^{2}\right]^{5 / 2}}
$$

As it has been derived for the rectangular Crystalline Beam model, similarly we can obtain the longitudinal angular response frequency (corrected for the transformation to the laboratory trame)

$$
\Omega_{\|}^{2}=\frac{Q^{2} e^{2} g_{\|}(w) n_{s}^{3 / 2}}{b^{3} \gamma^{2} m A}
$$

with the exception for the case the beam is made only of a string in which case

$$
\Omega_{\|}^{2}=\frac{Q^{2} e^{2} \xi()}{\lambda_{\|}^{3} \gamma^{5} m A}
$$

where

$$
g_{0}=4 \sum_{j=1}^{\infty} 1 / j^{3}=4 \times 1.2
$$

In order for the beam to be stable against longitudinal perturbations the longitudinal response frequency must be positive, that is the condition

$$
g_{\|}(w)>0
$$

must he salisfied. 
The plotting of the longitudinal response function $g_{\|}(w)$, given by $\mathrm{Eq} .(18)$, is similar to the one given in Figure 2 obtained for the case of a rectangular Crystalline Beam. The condition given by Eq. (22) is satisfied only for values of the compactmess parameter w smaller than a quantity which depends on the complexity of the equilibrium configuration.

We are interested in the case of a very dense beam with a large number $n_{h}$ of particles pe. shell. In this case, shells are separated from each other and most of the interaction of a particle is with the neighboring ones on the same shell, as shown in Figure 4. We shall refer to this as the case of local-shell interaction. It applies when $n_{h} \gg 4 \pi n_{s}$. For this case, the summation at the right side of Eq. (18) simplifies since one retains only the $j=i$ terms. Moreover, the most significant contributions are due to the terms with either $j_{2}=0$ or $j_{3}=0$, that is from those particles aligned either longitudinally or azimuthally with the test particle. In good approximation then we have

$$
a_{\|} \|(w)=g_{0}\left(1 / w^{3}-1 / 2 i^{3 / 2} \theta^{3}\right)
$$

The longitudinal stability condition is to hold for any shell. It is seen that the innermost shell is the most fragile one, so that the stability criterion equivalent to Eq. (22) is simply

$$
w<1.3 \theta
$$

A more accurate nurierical calculation of Eq. (18) shows that the proportionality coefficient is actually 1.6 instead of 1.3 . This condition is equivalent to requiring that the beam is more compact longitudinally than along the azimuthal direction on the shell. This can be understood with the same type of arguments given at the end of Section 2.

A special case is a single String. In this case $g_{\|}(w)=g_{0} / w^{3}$ given by Eq. (21). This quantity is always positive, so that the Siring is the first configuration to appear and it is always longitudinally stable. (See Appendix A for more details).

The result we have found are consistent with those also obtained for the rectangular Crystalline Beam. In the following we shall assume a longitudinally compact Crystalline Beam for which the compactness parameter $w$ is sufficiently small. The beam does not need confinement and it is stable in the direction of motion (the longitudinal direction).

The beam nevertheless needs to be contined transversely for two reasons: first to preserve the transverse equilibrium configuration and second to provide stability of motion in the transverse plane. Both of these functions can be accomplished by adding an external restoring force which also varies linearly with the position within the beam. 


\section{Transverse Confinement of the Cylindrical Beam}

We shall still ascume a round beam with $b_{1}=b_{2}=b$. Let us describe the motion of the $i$ - th particle in the transverse plane by letting $r_{\perp i}=r_{0 \perp i}+q u$ where $r_{0 \perp i}$ is given by Eq. (10) corresponding to the position within the equilibrium configuration, and the second term is a radial perturbation assumed to be small, that is $q<<b_{i}$, and $\boldsymbol{u}=(\cos \xi$, $\sin \xi, 0)$, a constant vector.

Taking again the frame with the beam at rest, the equation of motion is, after linearization,

$$
\begin{aligned}
m A \ddot{\boldsymbol{r}}_{\perp i} & =Q e \boldsymbol{E}_{\perp i}\left(\boldsymbol{r}_{\perp i}\right) \\
& =Q \epsilon \boldsymbol{E}_{\perp i}\left(\boldsymbol{r}_{0 \perp i}\right)+\left.Q e \frac{d \boldsymbol{E}_{\perp i}\left(\boldsymbol{r}_{\perp i}\right)}{d \boldsymbol{r}_{\perp i}}\right|_{\boldsymbol{r}_{0 \perp i}} ^{q u}+\ldots . \\
& =m A \ddot{\boldsymbol{r}}_{0 \perp i}+m A q \ddot{\boldsymbol{u}}
\end{aligned}
$$

This can be broken down in two equations

$$
m A \ddot{\boldsymbol{r}}_{0 \perp i}=Q e \boldsymbol{E}_{\perp i}\left(\boldsymbol{r}_{0 \perp i}\right)
$$

and

$$
m A \ddot{q}=\left.Q e \frac{d \boldsymbol{E}_{\perp i}\left(\boldsymbol{r}_{\perp i}\right)}{d \boldsymbol{r}_{\perp i}}\right|_{\boldsymbol{r}_{() \perp i}} q
$$

The electric field is made of two contributions: the internal, due to the beam proper, and the equivalent external due to the restoring forces

$$
\boldsymbol{E}_{\perp i}\left(\boldsymbol{r}_{\perp i}\right)=\boldsymbol{E}_{\perp i}{ }^{(i n t)}\left(\boldsymbol{r}_{\perp i}\right)+\boldsymbol{E}_{\perp i}{ }^{(e x t)}\left(\boldsymbol{r}_{\perp i}\right)
$$

where

$$
E_{\perp i}{ }^{(e x t)}\left(\boldsymbol{r}_{\perp i}\right)=-k_{e x t} \boldsymbol{r}_{\perp i}
$$

and

In particular

$$
E_{\perp i}{ }^{(\text {int })}\left(\boldsymbol{r}_{\perp i}\right)=Q e \Sigma_{j \neq i} \frac{\boldsymbol{r}_{\perp i}-\boldsymbol{r}_{\perp j}}{\boldsymbol{r}_{i}-\boldsymbol{r}_{j} \boldsymbol{l}^{3}}
$$

$$
E_{\perp i}\left(r_{0 \perp i}\right)=\left(k-k_{e x t}\right) r_{0 \perp i}
$$

where $k$ is given by Eq.s (11) and (12) with $b_{1}=b_{2}=b$.

The transverse confinement condition is to be satisfied

$$
k_{\text {ext }}=k
$$

Since $k>0$ then it follows that $k_{\text {ext }}>0$ is also required. 


\section{The Storage Ring as a Betatron}

The continement condition can be satisfied hy having the heam circulating in a Betatron magnet which provides focusing of motion in both transverse directions at the same time 151. Nevertheless this introduce the complication of the curvarure, an issue which we shall discuss in a following paper. For the moment let us assume a Betarron magnet as the device providing the restoring loree.

We can identify the axis 1 with the horizontal direction $(x)$ lying on the plane of symmetry of the Betatron magnet and the axis 2 with the vertical direction ( $y$ ) perpendicular to it. The ma, netic field distribution is described by a field index $n$ and the components are

$$
\begin{aligned}
& \mathrm{B}_{y}=\mathrm{B}_{0}(1-n x / \rho) \\
& \mathrm{B}_{x}=-\mathrm{B}_{0} n y / \rho
\end{aligned}
$$

where $\rho$ is the bending radius and $B_{0}$ is the value of the bending field on the central circular orhit. It is easily seen that

$$
\begin{aligned}
k_{e . x t} & =\beta \mathrm{B}_{0}(1-n) / \rho & \text { in the horizontal direction } \\
& =\beta \mathrm{B}_{0} n / \rho & \text { in the vertical direction }
\end{aligned}
$$

To provide stahility of motion in hoth transverse directions it is required that the field index () $<n<1$. In general, for an elliptical heam, the confïnement condition from Eq. (32) can also he wrillen as $(i=1,2)$

$$
A \beta^{2} \gamma^{2} \pi \alpha(\eta) n_{\mathrm{i}} b_{\mathrm{i}}^{2}=Q^{2} r_{0} N \rho
$$

where $n_{1}=1-n$ and $n_{2}=n$. For a round beam $n=0.5$ is required.

\section{Transverse Stability of the Cylindrical Beam}

From symmetry considerations, it can be shown that the radial perturhation deses not induce a longitudinal component of the field. Moreover, the induced transverse tield is independent of the longitudinal location. Again, without affecting generality, we can chose the particle to which the perturbation is added corresponding to $i=(i, 0,())$. Thus

$$
\left.\frac{d E_{\perp i}\left(r_{\perp i}\right)}{d r_{\perp i}}\right|_{r_{(), i}}=-k_{\ell x t}-Q e g_{\perp}(w) n_{s}^{3 / 2} / b^{3}
$$

where $g_{\perp}(w)=-b_{\|}(w) / 2$ is the transverse response finction. In the range of $w$ where the longitudinal stability applies $g_{\perp}(w)<0$.

We have thus recovered a result that we have already obtained lor the case of the rectangular Crystalline Bedm. It is then obvious that the same considerations about heam stability also apply to the cylindrical heam. 
The stability of motion in the transverse plane is investigated by solving Eq. (27) taking into account Eq. (36). The following transverse stability condition is then derived

$$
k_{\text {ext }}>Q e w g_{\|}(w) n_{s}^{3 / 2} / 2 b^{3}
$$

which, when combined with Eq.(32) is equivalent to

$$
4(N / L) b>\gamma g_{\|}(w) n_{s}^{3 / 2}
$$

Let $N$ be the total number of particles in the beam, and let $L=2 \pi \rho$. 'T he Crystalline Beam is made of $N_{\|}$slices where $N_{\|}=L / \lambda_{\|}$. The number $N_{\perp}$ of particles per slice is $N_{\perp}=n_{s} n_{h}$. The total number of particles is $N=N_{\|} N_{\perp}$. The condition of confinement for transverse stability then becomes

$$
w g_{\|}(w)<4 n_{h}
$$

When this condition is combined with the one for the longitudinal stability, Eq.(24), a very narrow range for the compactness parameter $w$ is derived. (See Appendix B).

Define

$$
\varepsilon(w)=1-w g_{\|}(w) / 4 n_{h}
$$

which for stability will range between 0 and 1 . If the beam is stable, particles perform transverse oscillations with angular frequency (in the laboratory frame, for a round beam) given by

$$
\Omega_{\perp}^{2}=\frac{2 Q^{2} e^{2}(N / L)}{m A \gamma^{3} b^{2}} \varepsilon(w)
$$

\section{Conditions for Beam Crystallization}

With a perturbation added the beam will perform stable longitudinal oscillations provided that the amplitude $a_{\|}$of the oscillations is sufficiently small. The beam momentum spread is then measured by the maximum velocity encountered during the oscillations, that is

$$
\Delta p_{\|}=m A \gamma a_{\|} \Omega_{\|}
$$

where $\Omega_{\|}$is given by Eq.(19) or (20). Since the condition for crystallization can be taken as

$$
a_{\|}<\lambda_{\|}
$$

the same condition can be expressed in terms of the relative momentum spread as

$$
\Delta p_{\|} / p<\lambda_{\|} \Omega_{\|} / \beta c
$$

A similar condition ought also to be satisfied for the transverse momentum spread, that is

$$
\Delta p_{\perp}<m A \gamma \lambda_{\perp} \Omega_{\perp}
$$

with $\Omega_{\perp}$ given by Eq.(41). This condition is obtained by requiring that transverse oscillations have an amplitude

$$
a_{\perp}<\lambda_{\perp}=2 \pi b / n_{h}
$$




\section{Insertion of Drift Spaces}

Drifts are required for beam manipulation like injection, abort and cooling. The betatron magnet will thus he broken in $M$ identical periods each of length $\ell_{B}=2 \pi \rho / M$ and separated by drifts of length $\ell_{\mathrm{D}}$. The insertion of drifts will disrupt the equilibrium configuration and it will not be possible to maintain the beam cross-section constant. The beam size in the sector magnet will start larger to compensate for the beam growth in the subsequent drift as shown in Figure 6.

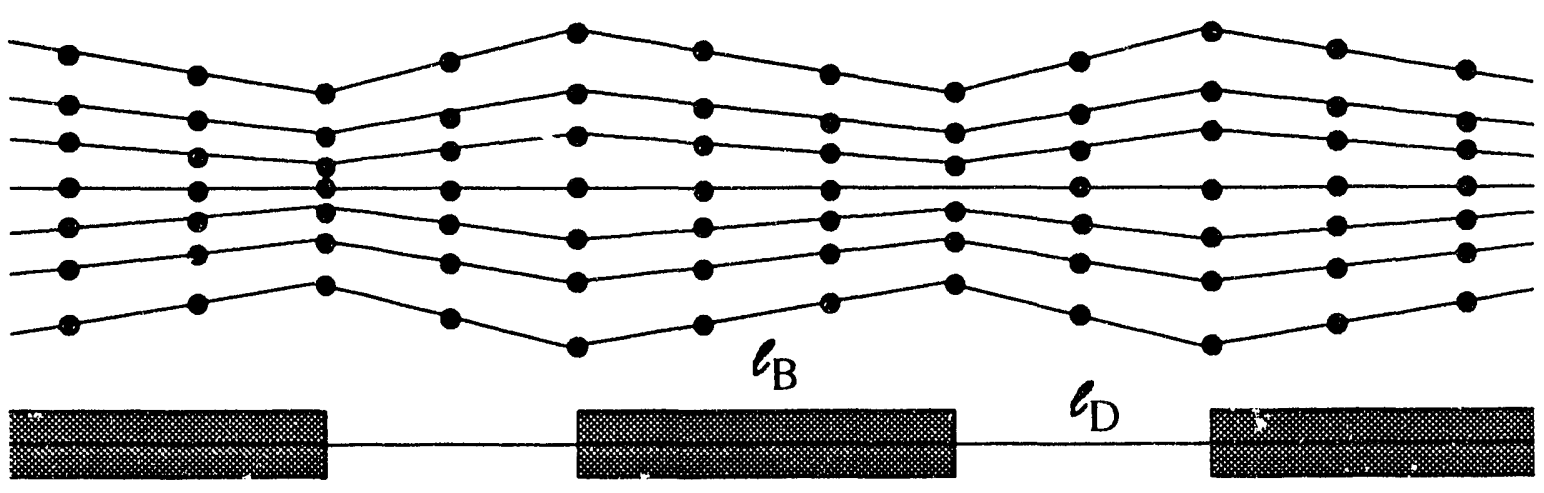

Fig. 6. Crystalline Beam in the presence of Drifts

Particle trajectories will acquire a pulsating behavior. The motion remains periodic with the period length equal to $2 \pi \rho / M+\ell_{1}$ ).

Let $z_{j}$ denote either the horizontal or the vertical coordinate of the $j$-th particle. It is more convenient to replace the time as the independent variable with the curvilinear length $s$ travelled along the reference closed orbit. The equation of motion can now be written as

$$
z_{j}^{\prime \prime}+\left[Q e\left(k_{\mathrm{ext}}-k\right) / m A \gamma \beta^{2} c^{2}\right] z_{j}=0
$$

where $k_{\mathrm{ext}}=0$ in the drifts and takes a constant value in the sector magnets which is proportional to $1-n$ in the horizontal plane and proportional to $n$ in the vertical plane.

For convenience let us write

$$
K=Q e k_{\mathrm{ext}} / m A \gamma \beta^{2} c^{2}
$$

The term $k$ which is proper of the beam itself, includes a dependence on the beam size $b$ and therefore will also vary periodically. It is convenient to write the dependence with the beam size explicilly

$$
Q_{e k} / m A \gamma \beta^{2} c^{2}=\frac{2 Q^{2} e^{2}(N / L)}{m A \gamma^{3} b^{2} \beta^{2} c^{2}}=h / b^{2}
$$

The solution of the equation above of course has to be periodic with the period given by $\ell_{B}+\ell_{D}$. In particular, the equation of motion can be written for the particle at the edge of the beam with $z_{j}=b$, obtaining in this way the equation of the beam envelope

$$
b^{\prime \prime}+K b-h / b=() \text {. }
$$

We shall assume that the drifts are not too long so that the beam dimension remain almost constant with a litte periodic modulation added. Moreover we shall assume that the heam 
is round or very close to roundness so that we can ignore the $\alpha(\eta)$ function and treat the hehavior of the two transverse dimensions essentially independent from each other.

Let $b=b_{0}(1+\Delta)$ where $b_{0}$ is the beam size in absence of drifts. We shall expand and retain only terms linear in $\Delta$. In the drift regions $K=0$ and the envelope equation reduces to

$$
\Delta^{\prime \prime}+\left(h / b_{0}^{2}\right) \Delta=h / b_{0}^{2}
$$

The solution of this equation can be represented with matrix notation. Let the vector $\left(\Delta, \Delta^{\prime}, 1\right)_{1}$ represent the status of motion at the entrance of a drift and let $\left(\Delta, \Delta^{\prime}, 1\right)_{2}$ represent the same at the exit. The two vectors are related to each other by the $3 \times 3$ transfer matrix

$$
\mathrm{M}_{\mathrm{D}}=\left|\begin{array}{ccc}
\cos \theta_{\mathrm{D}} & \left(\sin \theta_{\mathrm{D}}\right) / \sqrt{K_{\mathrm{D}}} & 1-\cos \theta_{\mathrm{D}} \\
-\sqrt{K_{\mathrm{D}}} \sin \theta_{\mathrm{D}} & \cos \theta_{\mathrm{D}} & \sqrt{K_{\mathrm{D}}} \sin \theta_{\mathrm{D}} \\
0 & 0 & 1
\end{array}\right|
$$

where $K_{\mathrm{D}}=h / b_{0}^{2}$ and $\theta_{\mathrm{D}}=\ell_{\mathrm{D}} \sqrt{K_{\mathrm{D}}}$ and $K_{\mathrm{D}}$ is a positive quantity.

The equation for the sector magnet, taking into account that $K=h / b_{0}^{2}$, and denoting with $K_{\mathrm{B}}=K+K_{\mathrm{D}}$ a positive quantity, is simply

$$
\Delta^{\prime \prime}+K_{\mathrm{B}} \Delta=0
$$

The transfer matrix associated to the sector magnet can be written as

$$
\mathrm{M}_{\mathrm{B}}=\left|\begin{array}{ccc}
\cos \theta_{\mathrm{B}} & \left(\sin \theta_{\mathrm{B}}\right) / \sqrt{K_{\mathrm{B}}} & 0 \\
-\sqrt{K_{\mathrm{B}}} \sin \theta_{\mathrm{B}} & \cos \theta_{\mathrm{B}} & 0 \\
0 & 0 & 1
\end{array}\right|
$$

where $\theta_{\mathrm{B}}=\epsilon_{\mathrm{B}} \sqrt{K_{\mathrm{B}}}$.

The transfer matrix for a period beginning with a drift is

$$
M_{B D}=M_{B} M_{D}
$$

and the one beginning with a sector magnet

$$
\mathrm{M}_{\mathrm{DB}}=\mathrm{M}_{\mathrm{D}} \mathrm{M}_{\mathrm{B}}
$$

Let either one of the matrices be written as

$$
\left|\begin{array}{ll}
m & \varepsilon \\
0 & 1
\end{array}\right|
$$

where $M$ is a $2 \times 2$ matrix of elements $m_{\mathrm{ij}}$ and $\varepsilon$ a 2 -element column vector of elements $e_{1}$ and $e_{2}$ which can be easily derived. The beam size is obtained by requiring that 


$$
m\left|\begin{array}{l}
\Delta \\
\Delta^{\prime}
\end{array}\right|+\varepsilon=\left|\begin{array}{l}
\Delta \\
\Delta^{\prime}
\end{array}\right|
$$

which can be solved to give

$$
\begin{aligned}
\Delta & =\frac{e_{2} m_{12}-e_{1}\left(m_{22}-1\right)}{2-\operatorname{Tr} m} \\
\Delta^{\prime} & =\frac{e_{1} m_{21}-e_{2}\left(m_{11}-1\right)}{2-\operatorname{Tr} m}
\end{aligned}
$$

Obviously it is very important to avoid that $\operatorname{Tr} m$ is too close to 2 . The only acceptable solutions are those with $|\Delta|<1$. These quantities are easily calculated on a computer.

\section{Stability with Drift Spaces inserted}

The new equilibrium configuration with drifts inserted should also be checked for stability. The longitudinal stability is automatically satisfied with the exception that now the longitudinal response angular frequency $\Omega_{\|}$has a modulation added due to the dependence on the transverse crystal dimension.

To determine the stability on the transverse plane, as usual, we add a small perturbation $u_{j}$ to the $j$-th particle along one of the two transverse directions. Alter linearization, the equation of motion is

$$
u_{j}^{\prime \prime}+4 u_{j}=0
$$

where, in the sector magnets,

$$
q=\Omega_{\perp}^{2} / \beta^{2} c^{2}=4 B
$$

and, in the drifts,

$$
\left.q=-q_{B} w g_{\|}(w) / 4 n_{h} \varepsilon(w)=-q_{1}\right)
$$

As usual, we can represent the solution with matrix notation. Only $2 \times 2$ matrices are required. The transfer matrix for the sector magnet is

$$
T_{B}=\left|\begin{array}{cc}
\cos \xi_{B} & \left(\sin \xi_{B}\right) / \sqrt{q_{B}} \\
-\sqrt{q_{B}} \sin \xi_{B} & \cos \xi_{B}
\end{array}\right|
$$

where $\xi_{B}=\ell_{B} \sqrt{q_{B}}$. The transfer matrix corresponding to the dritt is

$$
T_{1)}=\left|\begin{array}{cc}
\left(\cosh \xi_{1}\right) & \left(\sinh \xi_{1}\right) / \sqrt{q_{1}} \\
\left.\sqrt{\left.q_{1}\right)} \cdot \sinh \xi_{1}\right) & \left(\cosh \xi_{1)}\right.
\end{array}\right|
$$

where $\xi_{1)}=\varphi_{1} \sqrt{4(1)}$ 
The motion is stable if

$$
-2<\operatorname{Tr}\left\{T_{B} T_{D}\right\}<2
$$

which can be written explicitly as follows

$$
-2<2 \cos \xi_{\mathrm{B}} \cosh \xi_{\mathrm{D}}+\left(\sqrt{q_{\mathrm{D}} / q_{\mathrm{B}}}-\sqrt{q_{\mathrm{B}} / q_{\mathrm{D}}}\right) \sin \xi_{\mathrm{B}} \sinh \xi_{\mathrm{D}}<2
$$

A similar condition is also to be satisfied for the stability of motion in the storage ring in the limit of zero space-charge forces, that is

$$
-2<2 \cos \left(\ell_{\mathrm{B}} / \rho\right)+\left(\ell_{\mathrm{D}} / \rho\right) \sin \left(\ell_{\mathrm{B}} / \rho\right)<2
$$

\section{An Example of Crystalline Beam}

The following example has been worked out for the CRYSTAL project [3] where a beam of ions partially stripped is injected into a small storage ring from the Tandem-ALPI complex at the Laboratori Nazionali di Legnaro. The beam has the following characteristics:

$\begin{array}{lcl}\text { Charge State, Q } & 51 & \\ \text { Mass Number, A } & 197 & \\ \text { Rest Energy } & 931.15 & \mathrm{MeV} / \mathrm{u} \\ \text { Magnetic Rigidity } & 1.2 & \mathrm{~T}-\mathrm{m} \\ \text { Kinetic Energy } & 4.59 & \mathrm{MeV} / \mathrm{u} \\ \beta & 0.09893 & \end{array}$

The injector complex can provide an average beam current of $3 \mu \mathrm{A}$-particle with an emittance of about $1 \pi \mathrm{mm}$ mrad and a momentum spread $\Delta \mathrm{p} / \mathrm{p} \sim 0.01 \%$.

\begin{tabular}{|c|c|c|}
\hline No. of Periods & 4 & \\
\hline Bending Field & 3 & $\mathrm{kG}$ \\
\hline Bending Radius & 4 & $\mathrm{~m}$ \\
\hline Magnet Length & 6.283 & $\mathrm{~m}$ \\
\hline Bending Angle & 90. & degrees \\
\hline Drift Length & 2.0 & $\mathrm{~m}$ \\
\hline Circumference, $2 \pi R$ & 33.133 & $\mathrm{~m}$ \\
\hline Revolution Frequency & 0.895 & $\mathrm{MHz}$ \\
\hline
\end{tabular}

The storage ring is made of 4 periods. Each period includes a bending magnet with a field profile of index $n=0.5$, and a drift space. The ring parameters are as follows:

The drift length has been determined as the maximum value allowed before causing considerable disruption to the equilibrium configuration of the Crystalline Beam. The lattice of the storage ring for the diluted initial beam state is found to be stable, with a constant dispersion of $8 \mathrm{~m}$ and an amplitude lattice function $\beta_{\mathrm{L}}=6.22 \mathrm{~m}$. Also, the number of betatron oscillations per revolution in this case is 0.8156 .

Confinement of the crystalline form of the beam is maintained with an oscillation of the heam envelope of relative amplitude $\Delta=0.1122$. 
Stability is also insured for both longitudinal and transverse directions. The number of betatron oscillations per revolution for the dense state of the beam is now down to 0.3125 . The beam longitudinal response frequency is about $1 \mathrm{MHz}$ and the transverse response frequency is $0.5 \mathrm{MHz}$.

Figure 7 gives the total number $N$ of ions required in the storage ring for the formation of a Crystalline Beam with $n_{s}$ shells. It is seen that by increasing the intensity

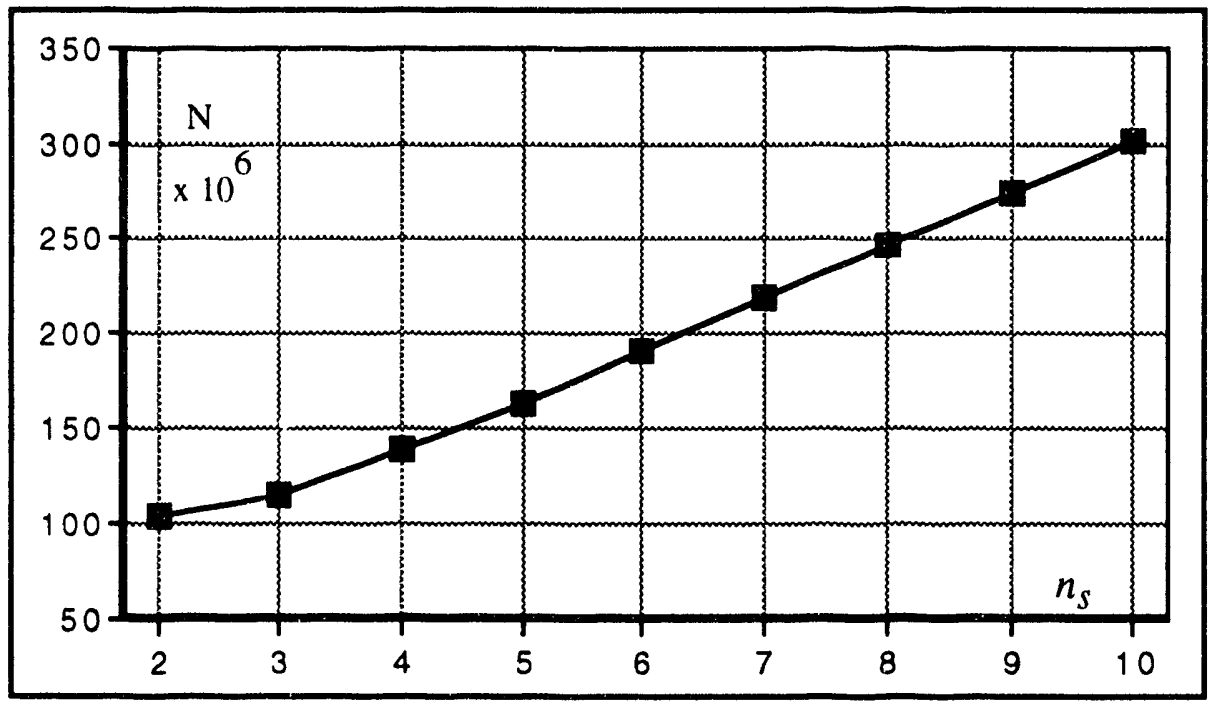

Fig. 7 Total Number of Ions vs. Number of $n_{s}$ shells

of the circulating beam it is possible to reach more complicate configurations with larger numbers of shells. Figure 8 is a plot of the number $n_{h}$ of ions per shell versus the number $n_{s}$ of shells. The variation of the longitudinal spacing $\lambda_{\|}$is plotted in Figure 9 , also versus the number $n_{s}$ of shells, whereas Figure 10 gives a similar plot for the beam radius $b$ in the final state. Finally, Figure 11 is the plot of the longitudinal and transverse beam momentum spread required for reaching the crystallization state.

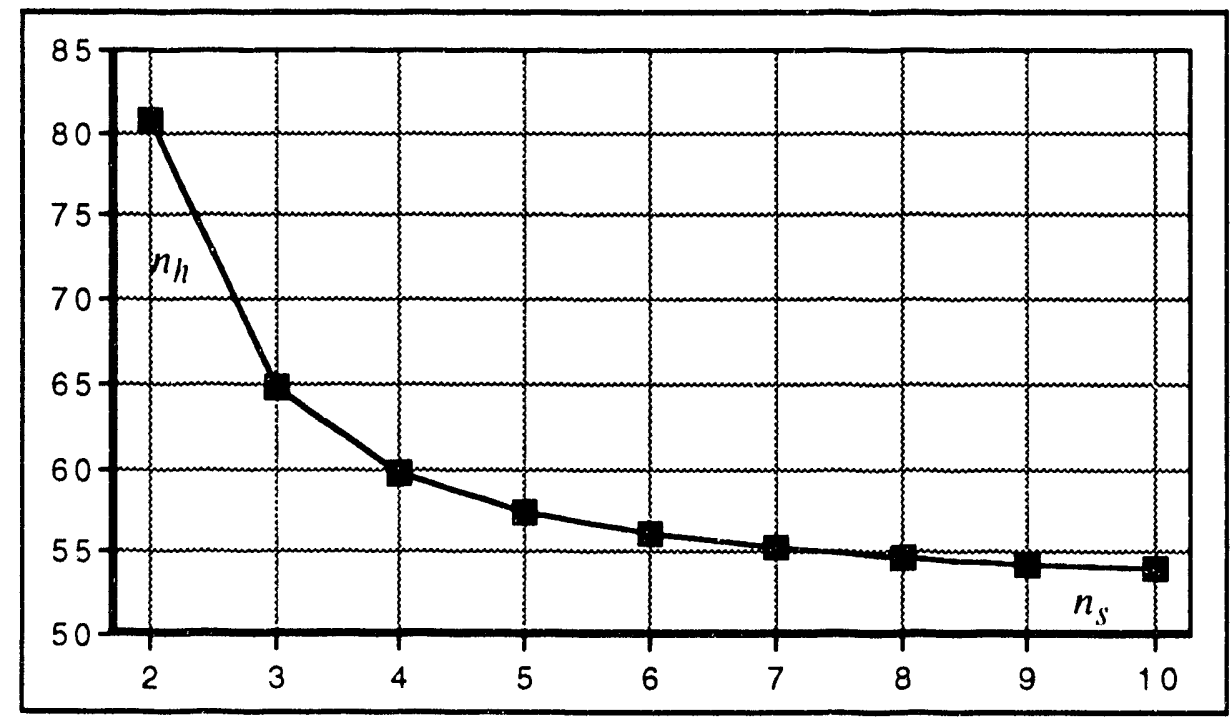

Fig. 8 Number $n_{h}$ of Ions per shell vs. Number $n_{s}$ of shell 


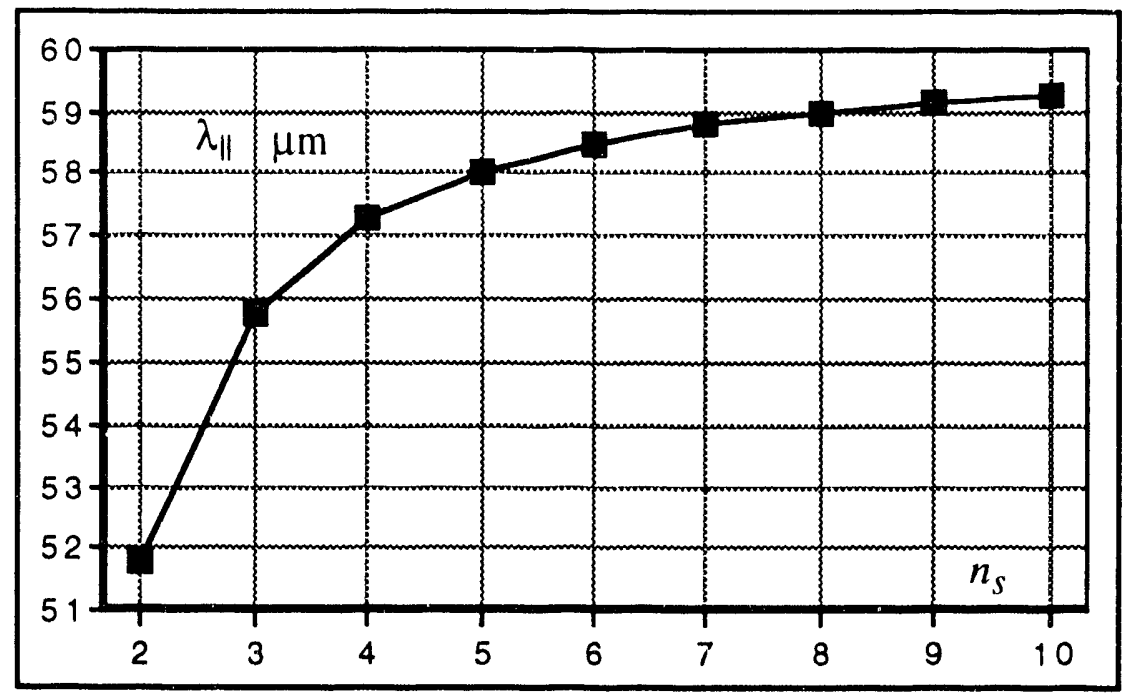

Fig. 9 The Longitudinal Spacing $\lambda_{\|}$vs. the Number $n_{s}$ of shells

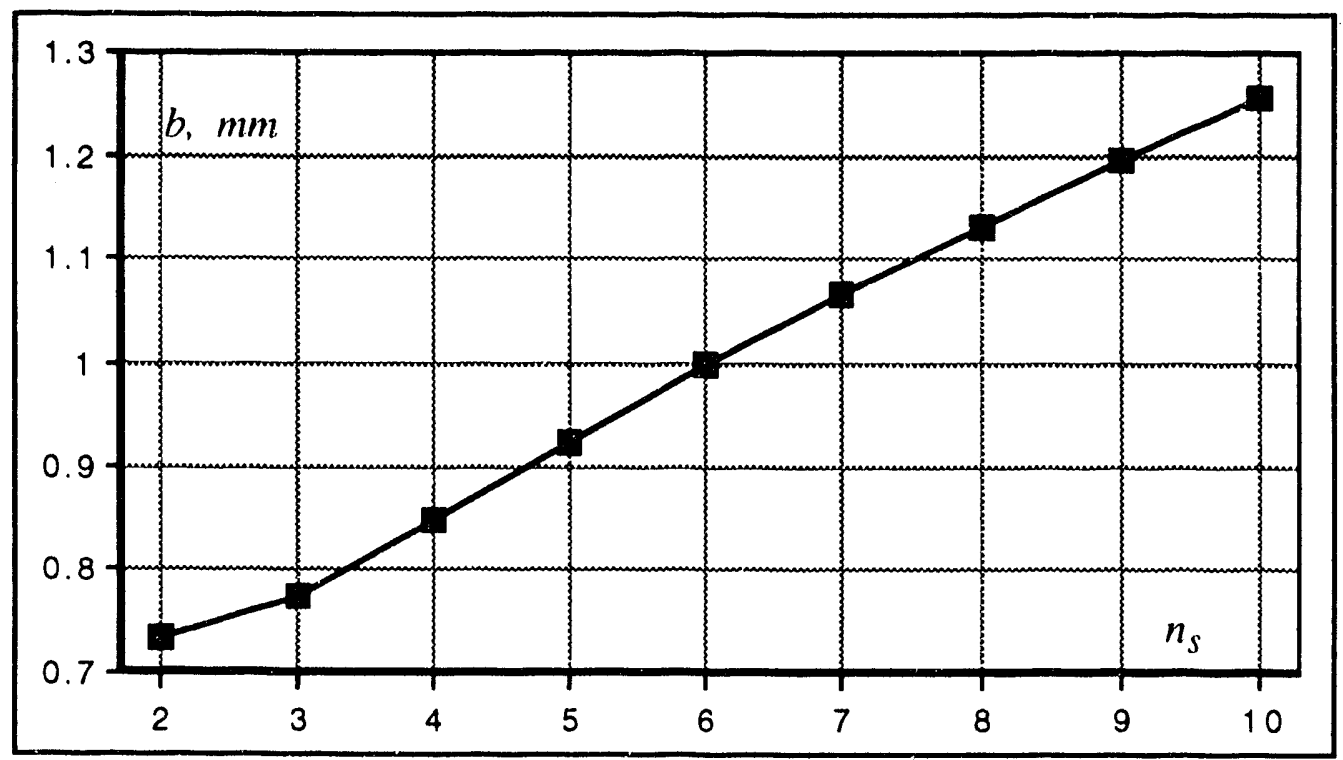

Fig. 10 The Beam Radius $b$ vs. the Number $n_{s}$ of shells

\section{Conclusions}

We have investigated in this report the fundamental properties of a Crystalline Beam circulating in a storage ring. The beam was delined by first determining its equilibrium configuration, corresponding to transverse space charge forces which vary linearly within the beam distribution. The second problem was to provide confinement of the equilibrium configuration. To avoid confinement in the direction of motion, we have found a criterion on the longitudinal compactness of the beam. Confinement in the transverse plane was then required with external restoring forces. A cylindrically symmetric beam can be obtained with focusing in both transverse dimensions; this can be done by adopting for the 
storage ring a magnet with a weak field profile added. Stability of the configuration was the third issue. This has to be insured with the same external restoring forces.

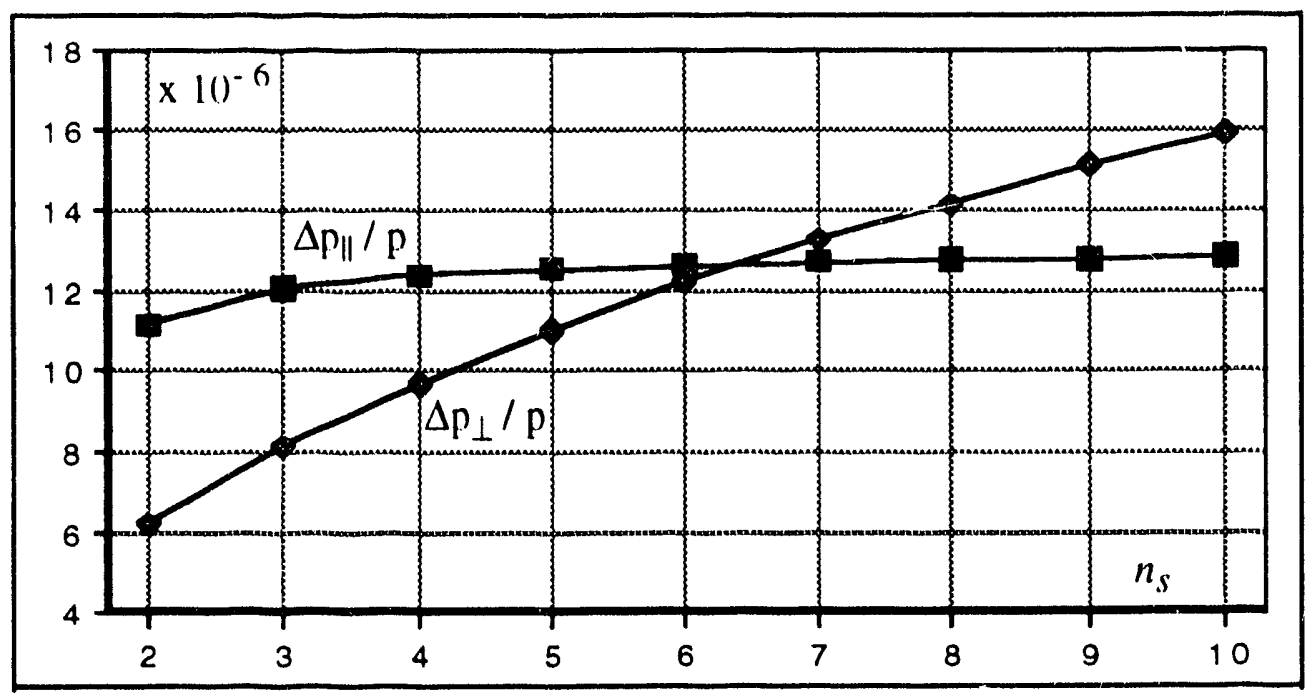

Fig. 11 Momentum Spreads for Beam Crystallization

We have also determined the disruptive effects of the insertion of drift spaces in the storage ring, and given an example of a storage ring where all the requirements are fulfilled. We have not discussed here the effect of the curvature due to the circular orbit in the bending magnet. We leave this topic to a subsequent paper.

\section{Acknowledgments}

The author of this report would like to show his appreciation for so many useful and informative discussions with the scientific staff of the Laboratori Nazionali di Legnaro near Padua in Italy. This work is a contribution to the CRYSTAL collaboration.

\section{References}

[1] J. P. Schiffer and A. Rahman, "Feasibility of a Crystalline Condensed State in Cooled Ion Beams of a Storage Ring", PHY-5121-ME-88, Feb. 1988, Argonne National Laboralory.

12| Proceedings of the Workshop on Crystalline Ion Beams, Werheim, Germany, oct. 1988. GSI-89-10 Report.

13) CRYSTAL. A Storage Ring for Ion Beam Crystallisation Studies. LNL-INFN (REP) 59/92 (1992). Laboratori Nazionali di Legnaro, Via Romea 4, 1-35(12() Legnaro (Padova), Italy

[4] A. G. Ruggiero, "Review of Space Charge Calculations", Booster Technical Note No. 1(14, January 1988. AD Department, Brookhaven National Laboratory.

15] E. Persico, E. Ferrari and S. E. Segre, "Principles of" Particle Accelerators", W. A. Benjamin, Inc. New York, Amsterdam (1968). 


\section{Appendix A: The String}

The string is a special arrangement which requires a more careful analysis.

The equilibrium configuration is given by locating all the particles on the longitudinal axis, equally spaced from each other by the distance $\lambda_{\|}$. Thus there are no transverse dimensions involved. If the heam is observed in its own rest frame, the $i$ - th particie is located at the longitudinal coordinate $z_{i}=\lambda_{\|} \gamma i$

This configuration obviously does not need confinement in either the longitudinal or the transverse direction.

Longitudinal stability is determined as usual by adding a small longitudinal perturbation $\lambda_{\|} \gamma u$, with $u<<1, t(0)$ any particle. Because of the arbitrary of the choice of the origin on axis, we can take the test particle to be located at the origin itself $(i=0)$. The expression of the longitudinal field acting on the test particle is calculated and linearized with respect to the perturbation

$$
E_{\|}=-Q e g_{0} u / \lambda_{\|}^{2} \gamma^{2}
$$

where $g_{0}$ is given by Eq. (21). The equation of motion is then written as follows

$$
A m \lambda_{\|} \gamma \ddot{u}=Q e E_{\|}
$$

and one determines that the particle performs stable longitudinal oscillations with the angular frequency $\Omega_{\|}$given by Eq. (20).

Stability of motion against transverse perturbation requires, on the contrary, some external restoring forces for focusing. Stability is determined by adding a lateral displacement $x \ll \lambda_{\|} \gamma$ to a test particle, which can be taken again as the one located at the origin of the axis $(i=0)$. The transverse electric field acting on the test particle is calculated and then linearized with respect to the particle displacement

$$
E_{\perp}=Q e g_{0} \times 12 \lambda_{\|}^{3} \gamma^{3}
$$

The equation of motion is then derived

$$
A m \ddot{x}=-Q e\left(k_{e x t} x-E_{\perp}\right)
$$

where $k_{e . x t}$ is the external restoring force parameter given by Eq. (34) with $n=0.5$. The transverse stability condition is then

$$
k_{e x t}>Q e g_{0} / 2 \lambda_{\|}^{3} \gamma^{3}
$$

If this condition is satisfied, particles will perform stable oscillations with the angular frequency $\Omega_{\perp}$ given by

$$
\Omega_{\perp}^{2}=\left(Q_{e} / A m \gamma^{2}\right)\left(k_{e x t}-Q_{e} g_{0} / 2 \lambda_{\|}^{3} \gamma^{3}\right)
$$

The stability of motion in the storage ring lattice with drifts is investigated with the same equations and matrix formalism described in Section 10 with the only exception that now

$$
q_{\mathrm{D}}=Q^{2} e^{2} g_{0} / 2 A m \beta^{2} \mathrm{c}^{2} \lambda_{\|}^{3} \gamma^{5}
$$




\section{Appendix B: Stability Ranges and Thresholds}

It is useful to investigate more in detail the conditions of longitudinal and transverse stahility as given by Eq.s (22) and (39). Let us make reference to Figure Bl which plots the function $w g_{\|}(w)$ versus the compaction parameter $w$ for $n_{s}=3$ in arbitrary units. The three curves correspond to each of the three shells which male the beam. It is clearly seen that, in order to satisty the longitudinal condition of Eq. (22), the compaction parameter $w$ is required to be smaller than a quantity $w_{\mathrm{L}}$ which depends on the shell number $i, i=1$ being the innermost and $i=n_{s}$ the outermost shell. From Eq. (23), which, as we have seen, is a good approximation of the response function $g_{\|}(w)$, we derive

$$
w_{\mathrm{L}}=2^{1 / 3} i^{1 / 2} \theta
$$

The motion is to be stable on all shells at the same time. Since the longitudinal stability condition is the most stringent for the innermost shell, we obtain then the overall condition for longitudinal stability, Eq. (24).

On the other hand, the transverse stability condition on a given shell corresponds to having the compaction parameter $w$ larger than a quantity $w_{\mathrm{T}}$ which also depends on the shell number $i$. Combining Eq.s (23) and (39) the following relation holds on any shell

$$
w_{\mathrm{T}}^{3} / w_{\mathrm{L}}^{3}+4\left(n_{h} / g_{0}\right) w_{\mathrm{T}}^{2}-1=0
$$

From construction, as shown in Figure B1, it is easily seen that $w_{\mathrm{T}}<w_{\mathrm{L}}$. Thus the general stability condition on a particular shell can be written as

$$
w_{\mathrm{T}}<w<w_{\mathrm{L}}
$$

It is also seen, again by construction, that the transverse stability condition is the most stringent on the outermost shell, that is for $i=n_{s}$. Thus the overall stability is obtained on a much restricted range which is also given by Eq. (B3) except that $w_{\mathrm{T}}$ is estimated at $i=n_{s}$ and $w_{\mathrm{L}}$ at $i=1$. A beam configuration has a stability threshold which occurs when the lower and upper stability limits coincide. The threshold condition is derived from Eq. (B2) by letting $w_{\mathrm{L}}=2^{1 / 3} n_{s}{ }^{1 / 2} \theta$ and $w_{\mathrm{T}}=2^{1 / 3} \theta$ which yields the following relation between $n_{s}$ and $n_{h}$

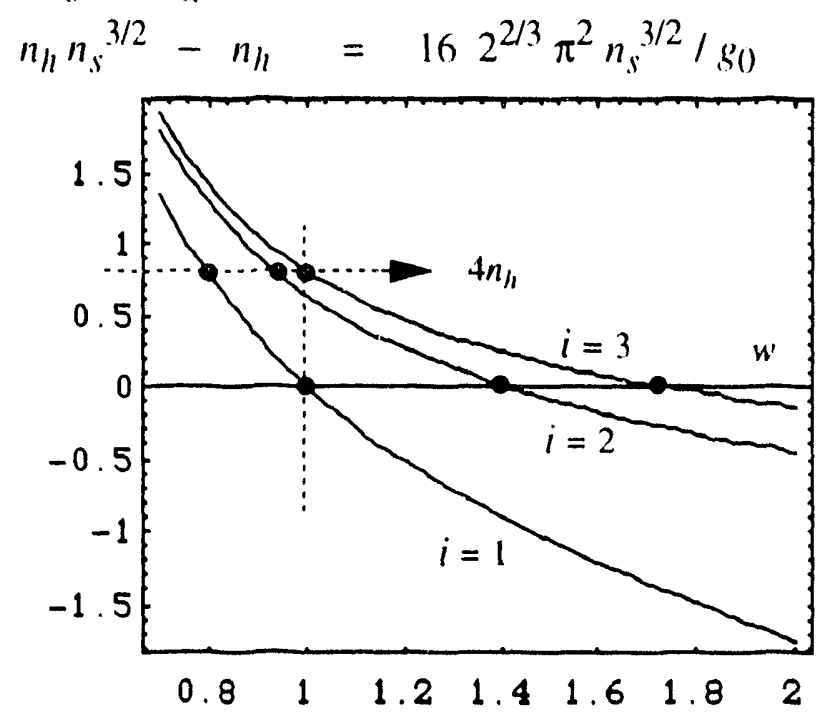

Fig. B I Response Functions $w g_{\|}(w)$ for $n_{s}=3$ shells 


\section{Appendix C: The Single Shell}

The stability threshold condition Eq. (B4) is valid for multi-shell beams, that is $n_{s}>1$. The case of a single shell, $n_{s}=1$, is also a special case. For it there is no stability threshold and the relation $w_{\mathrm{T}}<w_{\mathrm{L}}$, holds always with $w_{\mathrm{L}}=2^{1 / 3} \theta$ and $w_{\mathrm{T}}$ obtained by solving Eq. (B2). This is a cubic equation that in principle can be solved exactly; but since we expect that $w_{\mathrm{L}}$ and $w_{\mathrm{T}}$ are close $\mathrm{t}$ e each other, we can set $w_{\mathrm{T}}=(1-\delta) w_{\mathrm{L}}$ and treat $\delta$ small, in which case, after linearization of $\mathrm{Eq} .(\mathrm{B} 2)$, we derive

$$
\delta=\left(4 n_{h} / 3 g_{0}\right) w_{\mathrm{L}}^{2}
$$

$\delta w_{\mathrm{L}}$ represents the range of stability for a single-shell Crystalline Beam. 

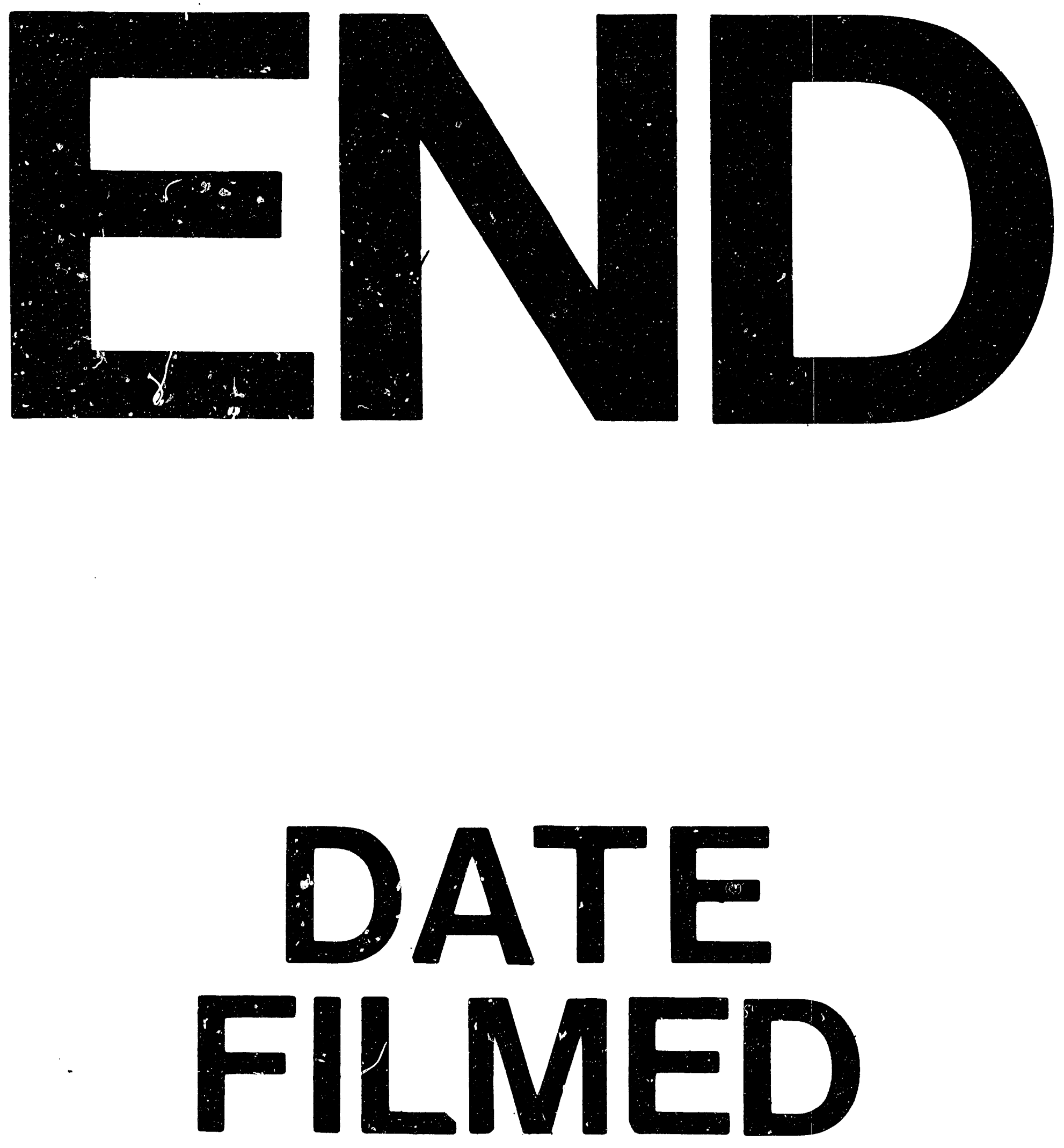

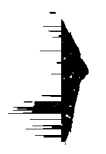

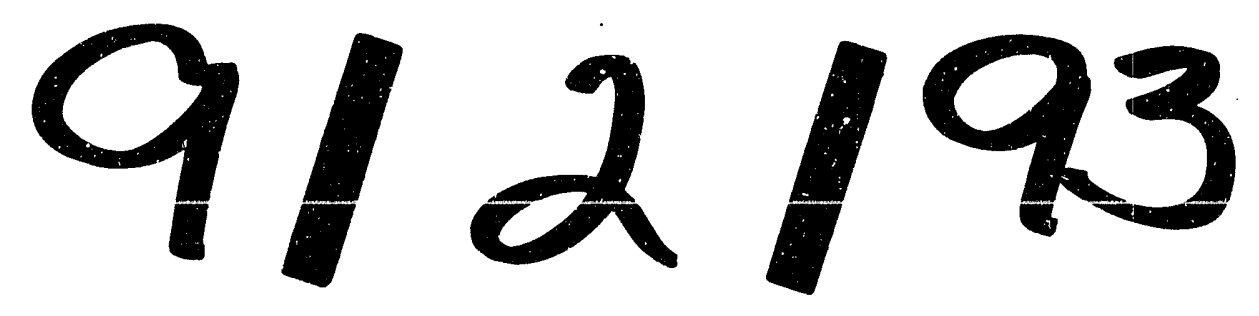


. 